\title{
Serpentes peçonhentas e ofidismo em Cruzeiro do Sul, Alto Juruá, Estado do Acre, Brasil
}

\author{
Paulo Sérgio BERNARDE ${ }^{1}$, Jáson de Oliveira GOMES²
}

\begin{abstract}
RESUMO
Apesar da importância dos acidentes ofídicos na Saúde Pública, são relativamente poucas as pesquisas realizadas sobre esse tema no Brasil. Devido aos poucos estudos sobre ofidismo na Amazônia e especialmente no estado do Acre, trabalhos epidemiológicos são de grande relevância. Esse estudo apresenta a lista de serpentes peçonhentas e aspectos epidemiológicos dos acidentes ofídicos em Cruzeiro do Sul, região do Alto Juruá (Acre), verificando quais gêneros de serpentes são responsáveis pelos envenenamentos e aspectos que envolvem o acidente e o atendimento hospitalar. Os dados epidemiológicos foram coligidos a partir do SINAN (Sistema de Informaçóes de Agravos de Notificação), no setor de vigilância epidemiológica do Hospital Regional do Juruá, no município de Cruzeiro do Sul. Sete espécies de serpentes peçonhentas foram registradas nesse município: três viperídeos (Bothrops atrox, Bothriopsis bilineata e Lachesis muta) e quatro elapídeos (Micrurus hemprichii, M. lemniscatus, M. remotus and M. surinamensis). Durante o período de dois anos (agosto de 2007 a julho de 2009) foram registrados 195 casos de acidentes ofídicos. Cinquenta e um por cento dos acidentes foi classificado como laquético (Lachesis), seguido pelo botrópico (Bothrops e Bothriopsis) com 38\% e crotálico (Crotalus) com 2\%. Em 9\% dos casos o gênero da serpente envolvida não foi informado. A maioria dos acidentes envolveu indivíduos adultos do gênero masculino em área rural, afetados principalmente nos membros inferiores. Os casos ocorreram mais frequentemente nos meses de novembro a abril, coincidindo com os maiores níveis pluviométricos. A maioria dos acidentes foi atribuída equivocadamente à serpente $L$. muta, tendo sido provavelmente causados por $B$. atrox.
\end{abstract}

PALAVRAS-CHAVES: Amazônia, Acidentes ofídicos, Envenenamento humano, Epidemiologia.

\section{Venomous snakes and ophidism in Cruzeiro do Sul, Alto Juruá, State of Acre, Brazil}

\begin{abstract}
Despite the importance of accidents involving snakebites, research on this theme in Brazil is relatively rare, especially in the Amazon region. Due to the poor number of studies about ophidism in the state of Acre and to the importance of this type of research in public health, epidemiological works on snake bite accidents are of great relevance. This study presents the list of species of poisonous snakes and the epidemiological aspects of snakebites in Cruzeiro do Sul, region of Alto Juruá (State of Acre, Brazil), in order to verify the snake genera responsible for the bites, and the main aspects involving the accidents and hospital attending. The epidemiologic data were collected from SINAN (National Databank of Major Causes of Morbidity) in the sector of epidemiologic vigilance of Hospital Regional do Juruá, in Cruzeiro do Sul. Seven species of poisonous snakes were recorded in Cruzeiro do Sul: three viperids (Bothrops atrox, Bothriopsis bilineata and Lachesis muta) and four elapids (Micrurus hemprichii, M. lemniscatus, M. remotus and M. surinamensis). During a two-year period (August 2007 to July 2009) 195 cases of snakebites were recorded. Fifty-one percent of the accidents were classified as lachetic (Lachesis), followed by bothropic (Bothrops and Bothriopsis) with 38\% and crotalic (Crotalus) with 2\%. In 9\% of cases the snake genus involved was not informed. The majority of the accidents involved adult males living in rural areas, mainly affected in the lower limbs. The cases occurred most frequently from November to April, coinciding with the highest pluviometric levels. Most snakebites were equivocally attributed to $L$. muta, and were probably caused by $B$. atrox.
\end{abstract}

KEYWORDS: Amazon, Snakebites, Human envenomation, Epidemiology.

1 Universidade Federal do Acre, Campus Floresta, Laboratório de Herpetologia, Cruzeiro do Sul, E-mail: SnakeBernarde@hotmail.com

2 Universidade Federal do Acre, Campus Floresta, Cruzeiro do Sul, E-mail: enfer-jason@hotmail.com 


\section{INTRODUÇÃO}

No Brasil existem 371 espécies de serpentes registradas (Bérnils 2010), das quais 55 pertencentes às famílias Elapidae (27 espécies) e Viperidae (28 espécies) são consideradas peçonhentas e podem causar envenenamentos em seres humanos. De acordo com o gênero de serpente, os acidentes são classificados em botrópico, crotálico, laquético e elapídico (Araújo et al. 2003). Com as recentes mudanças taxonômicas das serpentes peçonhentas (Fenwick et al. 2009), os gêneros estão agora distribuídos de acordo com o tipo de acidente da seguinte forma: botrópico (Bothrops, Bothropoides, Bothriopsis, Bothrocophias e Rhinocerophis), crotálico (Crotalus), laquético (Lachesis) e elapídico (Micrurus e Leptomicrurus).

Ocorrem por ano aproximadamente 20.000 casos de acidentes ofídicos no Brasil, sendo que é exatamente na Amazônia onde existe maior incidência de casos por habitantes (Araújo et al. 2003). Entretanto, em virtude das maiores dificuldades de acesso aos serviços de saúde nessa regiâo (Araújo et al. 2003), é de se esperar que haja subnotificaçấo dos acidentes ofídicos. Apesar da Amazônia apresentar maior incidência de acidentes ofídicos, a maioria dos estudos epidemiológicos sobre o ofidismo sempre foi desenvolvida na regiấo sudeste do Brasil (Bochner e Struchiner 2003).

Alguns estudos epidemiológicos sobre o ofidismo realizados na Amazônia foram desenvolvidos nos estados do Amazonas (Borges et al. 1999; Waldez e Vogt 2010), Amapá (Lima et al. 2009), Roraima (Nascimento 2000) e Acre (Moreno et al. 2005). A maioria dos casos registrados na Amazônia foi atribuída a acidentes botrópicos (67,5 a 95\%), sendo a serpente Bothrops atrox a responsável pela maioria dos envenenamentos (Campbell e Lamar 2004). Alguns desses estudos revelaram certo despreparo no atendimento dos casos de ofidismo em relaçáo ao tratamento, como, por exemplo, no estabelecimento da quantidade adequada de ampolas de soro antiofídico a ser ministrado (Borges et al. 1999; Moreno et al. 2005; Lima et al. 2009).

No Acre, o ofidismo foi estudado por Moreno et al. (2005) no sul do estado, com base nos casos atendidos em Rio Branco, registrando 144 vítimas de acidentes ofídicos no ano de 2002. No Alto Juruá, regiáo noroeste do Acre, Pierini et al. (1996) estudaram os acidentes em populaçóes que vivem em florestas (indígenas, extrativistas e ribeirinhas), observando uma alta incidência (13\% de casos na amostra estudada).

Apesar da importância como problema de saúde, existe uma carência de estudos sobre ofidismo na Amazônia, especialmente na regiấo do Alto Juruá (Acre). Em vista disso, o presente estudo tem como objetivo apresentar a lista de serpentes peçonhentas que ocorrem nessa regiáo, bem como os aspectos epidemiológicos do ofidismo no município de Cruzeiro do Sul.

\section{MATERIAL E MÉTODOS}

Foi realizado um estudo retrospectivo coletando informaçóes sobre casos de acidentes ofídicos referentes ao período de agosto de 2007 a julho de 2009 atendidos no Hospital Regional do Juruá, município de Cruzeiro do Sul (Acre) (Figura 1). Esse município apresentava cerca de 77.000 habitantes durante o período desse estudo (IBGE 2010). As principais atividades econômicas da região săo a produção de farinha de mandioca, o extrativismo (açaí, buriti), a agricultura (milho, arroz, banana) e a pesca. O clima da região é caracterizado como tropical quente e úmido, com temperatura média anual de $24^{\circ} \mathrm{C}$ (Ribeiro 1977), sendo o período de maio a outubro considerado o mais seco do ano. Durante o período deste estudo o município apresentou uma precipitação pluviométrica anual de $2.405 \mathrm{~mm}$. Os dados climáticos foram obtidos na Estação Meteorológica de Cruzeiro do Sul do Instituto Nacional de Meteorologia (INMET).

Os dados epidemiológicos obtidos foram coletados a partir das fichas do SINAN (Sistema de Informação de Agravos de Notificaçấo) no Setor de Vigilância Epidemiológica do Hospital Regional do Juruá e do Sistema de Notificação da Secretaria Municipal de Saúde do município de Cruzeiro do Sul. Foram verificadas as seguintes variáveis: mês de ocorrência, identificaçáo da serpente (tipo de acidente), local do acidente (zona urbana, peri-urbana e rural), município de ocorrência, dados da vítima (faixa etária, gênero e região anatômica atingida), tempo decorrido entre o acidente e o atendimento, número de ampolas utilizadas e tipo de soro no tratamento das vítimas. Para verificar uma possível relação entre o número de acidentes ofídicos mensais com a pluviosidade, foi utilizado o teste de correlação de Spearman.

As informaçôes sobre a ocorrência das espécies de serpentes peçonhentas na região de Cruzeiro do Sul foram obtidas com

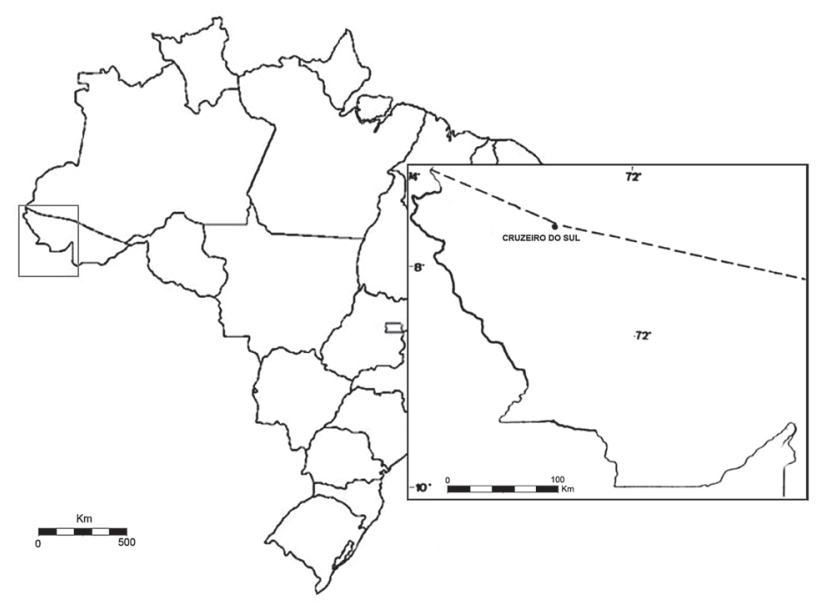

Figura 1 - Mapa do Brasil destacando a localização de Cruzeiro do Sul no Acre. 
base em consulta da Coleção Herpetológica da Universidade Federal do Acre, Campus Floresta, onde existem espécimes colecionados da região. Apresentamos também fotografias e nomes populares regionais dessas serpentes.

\section{RESULTADOS E DISCUSSÃO}

Em Cruzeiro do Sul e regiáo, ocorrem sete espécies de serpentes peçonhentas (Figuras 2 a 3): três viperídeos, a surucucu ou jararaca (Bothrops atrox), a papagaia (Bothriopsis bilineata) e a pico-de-jaca ou surucucu-pico-de-jaca (Lachesis muta), e quatro elapídeos, as corais-verdadeiras Micrurus hemprichii, $M$. lemniscatus, $M$. remotus e $M$. surinamensis. $\mathrm{O}$ juvenil de $B$. atrox é também conhecido como jararaquinhado-rabo-branco.

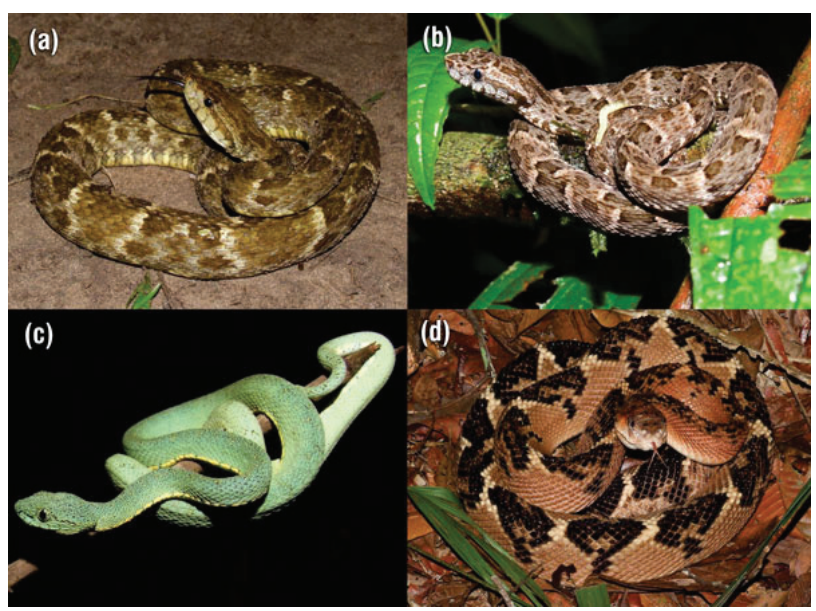

Figura 2 - Viperídeos que ocorrem em Cruzeiro do Sul: a) Jararaca ou Surucucu (Bothrops atrox); b) Jararaquinha-do-rabo-branco (Juvenil de B. atrox); c) Papagaia (Bothriopsis bilineata); d) Pico-de-jaca ou Surucucu-pico-de-jaca (Lachesis muta). Fotos por Paulo S. Bernarde.

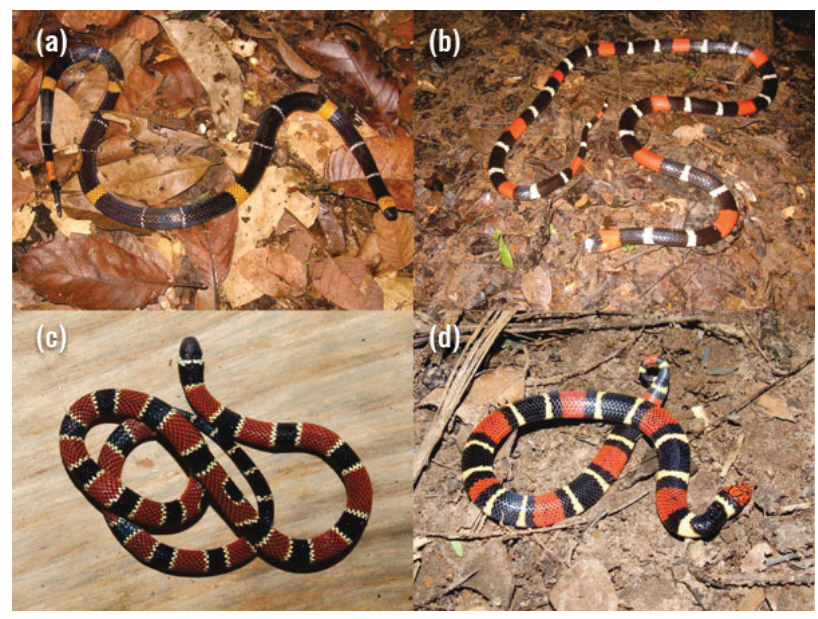

Figura 3 - Elapídeos (corais verdadeiras) que ocorrem em Cruzeiro do Sul: a) Micrurus hemprichii; b) Micrurus lemniscatus; c) Micrurus remotus; d) Micrurus surinamensis. Fotos por Paulo S. Bernarde.
No período de agosto de 2007 a julho de 2009 foram registrados 195 casos de acidentes ofídicos atendidos no Hospital Regional do Juruá em Cruzeiro do Sul (média de 126,62 casos por 100.000 habitantes por ano). Em todos os casos o diagnóstico do tipo de acidente (botrópico, laquético, crotálico ou elapídico) foi baseado em critérios clínicos, o que caracterizou o acidente como provável. Segundo os registros, a grande maioria dos acidentes $(51,3 \%)$ foi classificada como laquético (gênero Lachesis), seguido pelo botrópico (gêneros Bothrops e Bothriopsis) com 38\% e crotálico (gênero Crotalus) com $2 \%$ dos casos (Figura 4). Não foi registrado nenhum acidente por serpentes do gênero Micrurus. Registrou-se, ainda, 17 casos $(8,7 \%)$ onde o gênero da serpente envolvido não foi informado.

Quanto à sazonalidade, foi verificado que nos meses de maior pluviosidade (novembro a abril) houve uma maior quantidade de acidentes ofídicos (Figura 5), demonstrando uma correlaçáo positiva (Spearman $=0,6180 ; \mathrm{p}=0,0013 ; \mathrm{n}$ $=24)$.

Quanto à distribuição dos casos de ofidismo observou-se que a maioria dos acidentes ocorreu em pessoas do gênero masculino (76,4\%) com idades de 20 a 29 anos (Tabela 1). A regiáo anatômica mais atingida foi os membros inferiores $(86,1 \%)$, seguido dos membros superiores $(11,2 \%)$, cabeça $(0,5 \%)$ e tronco $(0,5 \%)$, e em $1,5 \%$ dos casos a região anatômica não foi informada. Foi registrado apenas um óbito ( $0,5 \%$ do total) atribuído a acidente laquético, sendo que a maioria dos casos (74\%) evoluiu para a cura e em $25 \%$ desses casos a evolução não foi informada.

A maioria dos acidentes $(70,2 \%)$ ocorreu na zona rural, seguida pela área urbana $(17,4 \%)$ e região peri-urbana $(9,2 \%)$, sendo que $3,1 \%$ teve como ignorado o local de ocorrência. Em relação ao tempo decorrido entre o acidente e o atendimento hospitalar, 53,6\% dos casos foram atendidos em menos de

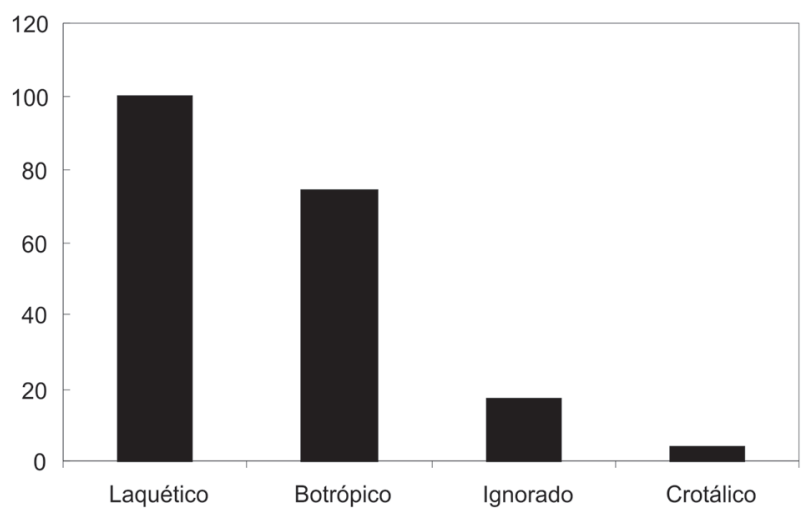

Figura 4 - Distribuição dos acidentes ofídicos atendidos no Hospital do Juruá em Cruzeiro do Sul (AC) de acordo com a serpente causadora durante agosto de 2007 a julho de 2009. 


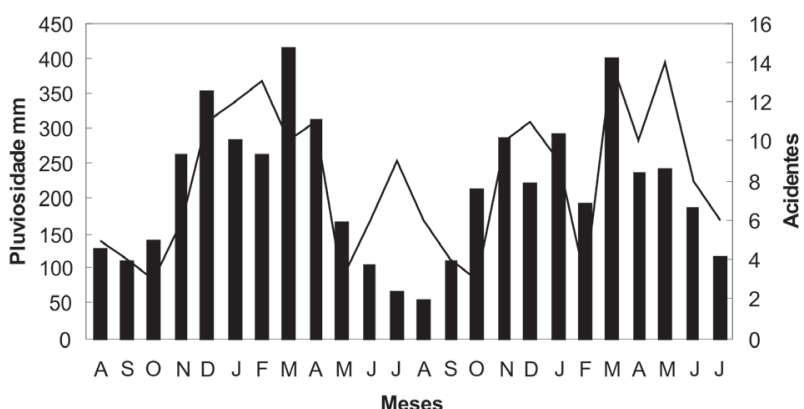

Figura 5 - Distribuição da pluviosidade (colunas) e dos acidentes ofídicos (linha) atendidos no Hospital do Juruá em Cruzeiro do Sul (AC) no período de agosto de 2007 e julho de 2009.

seis horas, $8,7 \%$ antes de 12 horas, $7,6 \%$ antes de 24 horas e $27,1 \%$ após 24 horas decorridas do acidente.

Segundo a gravidade, o número de acidentes botrópicos (Tabela 2) teve uma predominância de casos moderados (57), sendo que desses, em $64,9 \%$ foram administradas entre cinco e nove ampolas, em $24,5 \%$ foram administradas entre uma e quatro ampolas, e em $10,6 \%$ dos casos, entre 10 ou mais ampolas. Entre os acidentes considerados leves obteve-se um total de 13 casos, onde $84,8 \%$ receberam entre uma a quatro ampolas, um caso $(7,6 \%)$ recebeu sete ampolas e outro $(7,6 \%) 12$ ampolas. Em relação aos acidentes classificados como graves, ocorreu apenas um caso onde foram ministradas oito ampolas. A maioria dos pacientes $(64$ casos) recebeu soro antibotrópico, seguido de antibotrópico-laquético (4), antibotrópico-crotálico (2) e antilaquético (1).

Entre os acidentes considerados laquéticos (Tabela 3), a maioria $(92,6 \%)$ dos casos foi registrada como moderada. Desses, $75 \%$ receberam entre cinco e nove ampolas, 19,3\% entre uma e quatro ampolas, e 5,6\% entre 10 ou mais ampolas. Nos acidentes classificados como leves foram administradas entre uma e quatro ampolas. Apenas um caso foi considerado grave e recebeu oito ampolas. A maioria das vítimas (98 dos casos) foi tratada com soro antibotrópico-laquético, um caso com antibotrópico e outro com antilaquético.

Tabela 1 - Distribuição dos acidentes ofídicos segundo a faixa etária e gênero, na região do Juruá de agosto de 2007 a julho de 2009.

\begin{tabular}{lcccccc}
\hline \multirow{2}{*}{$\begin{array}{l}\text { Faixa etária } \\
\text { (anos) }\end{array}$} & \multicolumn{3}{c}{ Gênero } & \multicolumn{2}{c}{ Total } \\
\cline { 2 - 5 } & \multicolumn{2}{c}{ Masculino } & \multicolumn{2}{c}{ Feminino } & \multirow{2}{*}{$n^{0}$} & $\%$ \\
\cline { 2 - 5 } & $\mathrm{n}^{0}$ & $\%$ & $\mathrm{n}^{0}$ & $\%$ & & \\
\hline 0 a 9 & 13 & 76,5 & 04 & 23,5 & 17 & 8,7 \\
10 a 19 & 30 & 68,2 & 14 & 31,8 & 44 & 22,5 \\
\hline 20 a 29 & 48 & 81,3 & 11 & 18,7 & 59 & 30,2 \\
\hline 30 a 39 & 27 & 81,8 & 06 & 18,2 & 33 & 16,9 \\
\hline 40 a 49 & 15 & 78,9 & 04 & 21,1 & 19 & 9,7 \\
\hline$\geq 50$ & 16 & 69,5 & 07 & 30,5 & 23 & 11,8 \\
\hline Total & 149 & 76,4 & 46 & 23,6 & 195 & 100 \\
\hline
\end{tabular}

Tabela 2 - Distribuição dos acidentes ofídicos botrópicos (número de casos) segundo a quantidade de ampolas utilizadas e a gravidade do acidente.

\begin{tabular}{lcccccccc}
\hline & \multicolumn{2}{c}{$\begin{array}{c}1-4 \\
\text { ampolas }\end{array}$} & \multicolumn{2}{c}{$\begin{array}{c}5-9 \\
\text { ampolas }\end{array}$} & \multicolumn{2}{c}{$\begin{array}{c}10 \text { ou }+ \\
\text { ampolas }\end{array}$} & \multicolumn{2}{c}{ Total } \\
\cline { 2 - 11 } & $\mathrm{n}^{0}$ & $\%$ & $\mathrm{n}^{0}$ & $\%$ & $\mathrm{n}^{0}$ & $\%$ & $\mathrm{n}^{0}$ & $\%$ \\
\hline Leve & 11 & 84,8 & 1 & 7,6 & 1 & 7,6 & 13 & 100,0 \\
Moderado & 14 & 24,5 & 37 & 64,9 & 6 & 10,6 & 57 & 100,0 \\
Grave & 0 & 0,0 & 1 & 100 & 0 & 0,0 & 1 & 100,0 \\
\hline Total de casos & 25 & \multicolumn{2}{c}{39} & & 7 & & 71 \\
\hline
\end{tabular}

Tabela 3 - Distribuição dos acidentes ofídicos laquéticos (número de casos) segundo a quantidade de ampolas utilizadas e a gravidade do acidente.

\begin{tabular}{lcccccccc}
\hline & \multicolumn{2}{c}{$\begin{array}{c}1-4 \\
\text { ampolas }\end{array}$} & \multicolumn{2}{c}{$\begin{array}{c}5-9 \\
\text { ampolas }\end{array}$} & \multicolumn{2}{c}{$\begin{array}{c}10 \text { ou }+ \\
\text { ampolas }\end{array}$} & \multicolumn{2}{c}{ Total } \\
\cline { 2 - 10 } & $\mathrm{n}^{0}$ & $\%$ & $\mathrm{n}^{0}$ & $\%$ & $\mathrm{n}^{0}$ & $\%$ & $\mathrm{n}^{0}$ & $\%$ \\
\hline Leve & 6 & 100,0 & 0 & 0,0 & 0 & 0,0 & 6 & 100,0 \\
Moderado & 17 & 19,3 & 66 & 75,0 & 5 & 5,6 & 88 & 100,0 \\
Grave & 0 & 0,0 & 1 & 100,0 & 0 & 0,0 & 1 & 100,0 \\
\hline Total de casos & 23 & \multicolumn{2}{c}{67} & \multicolumn{2}{c}{5} & \multicolumn{2}{c}{95} \\
\hline
\end{tabular}

Os quatro acidentes registrados como crotálicos foram considerados moderados e os pacientes receberam 4, 5, 6 e 8 ampolas de soro. Três casos foram tratados com soro antibotrópico-crotálico e um com antibotrópico.

Dos casos em que a serpente causadora não foi determinada (21 casos), nove receberam soro antibotrópico-laquético, seis antibotrópico, um anticrotálico, um antibotrópico-crotálico e três nenhum tipo de soro.

Em Cruzeiro do Sul foi registrada uma alta incidência de acidentes ofídicos (126,62 casos por 100.000 habitantes), mais do que os coeficientes registrados para a Amazônia (28,6 casos/100.000 habitantes) e para o estado do Acre (26,3 casos/100.000 habitantes) (Moreno et al. 2005). Uma incidência alta de casos (127,6 a 237,3 casos/100.000 habitantes) também foi registrada em alguns municípios do interior do Amazonas por Santos (2003). Pierini et al. (1996) registraram uma alta prevalência de acidentes ofídicos nas populaçôes de seringueiros e indígenas que vivem nas florestas do Alto Juruá. Essa região conta com o menor índice de desmatamento do Acre (Silva e Ribeiro 2004). Esse fato deve estar associado principalmente à presença de florestas protegidas (um parque nacional, florestas estaduais, reservas extrativistas e terras indígenas) e às atividades extrativistas dos povos dessa regiáo (Almeida 2004). Esse fato faz com que os considerados "povos da floresta" estejam mais expostos aos acidentes ofídicos.

A maioria dos acidentes registrados foi atribuída à serpente Lachesis muta, fato que não era esperado já que essa espécie aparece como a de menor incidência nos registros da região amazônica (Borges et al. 1999; Nascimento 2000; Santos 2003; Málaque e França 2003; Campbell e Lamar 2004; 
Moreno et al. 2005; Lima et al. 2009). De acordo com estudos sobre ecologia de serpentes realizados na Amazônia, Bothrops atrox é a serpente peçonhenta mais abundante, enquanto que $L$. muta ocorre em menor densidade (Cunha e Nascimento 1993; Jorge-da-Silva 1993; Martins e Oliveira 1998; Oliveira e Martins 2001; Bernarde e Abe 2006; Turci et al. 2009). Em vista disso, presume-se que essa alta incidência de casos laquéticos em Cruzeiro do Sul esteja superestimada. A serpente $B$. atrox (jararaca) recebe o nome popular na regiâo do Alto Juruá de surucucu e esse mesmo nome popular em outras regióes do Brasil é atribuído para L. muta (conhecida no Alto Juruá e também em outras regiōes por pico-de-jaca), provavelmente causando dúvidas aos médicos no momento do diagnóstico quando o paciente declara qual foi a serpente causadora da picada. Sabe-se que uma espécie de serpente pode ter diferentes nomes populares ao longo de sua distribuição geográfica (Campell e Lamar 2004), o que pode gerar confusões. Waldez e Vogt (2010) observaram que ribeirinhos no baixo Rio Purus no Amazonas confundem indivíduos maiores de $B$. atrox com L. muta. Um estudo sorológico realizado pela técnica ELISA para diagnóstico do gênero de serpente causador de acidentes em Belém (PA) revelou que em 26 vítimas os envenenamentos foram causados por Bothrops, mas que $46,15 \%$ desses pacientes denominaram a serpente de surucucu (Pardal et al. 1997). Situação semelhante foi observada por Pierini et al. (1996), que ao testarem o sangue de vítimas de acidentes no Alto Juruá para detectarem a presença de anticorpos, verificaram que o gênero Bothrops foi responsável pela maioria dos acidentes $(86 \%)$. Essas informaçôes sustentam mais ainda a hipótese de que os acidentes laquéticos estejam superestimados em Cruzeiro do Sul, sendo que provavelmente grande parte desses casos tenha sido causada por $B$. atrox, a serpente peçonhenta mais abundante e mais amplamente distribuída pela Amazônia (Campbell e Lamar 2004).

Quatro acidentes foram registrados como crotálicos. Entretanto, as cascavéis (Crotalus durissus) na Amazônia estão presentes somente nas manchas de campos e cerrado em Vilhena (RO), Humaitá (AM), Ilha de Marajó, Santarém e Serra do Cachimbo (PA), no Amapá e Roraima (Melgarejo 2003; Campbell e Lamar 2004), estando ausentes no estado do Acre. Esse é, portanto, outro tipo de equívoco devido a diferenças nas aplicaçóes de nomes populares às serpentes pela população do Alto Juruá, onde algumas pessoas denominam a Lachesis muta de cascavel.

Apesar da regiáo norte ser caracterizada como não tendo uma sazonalidade marcante nos acidentes ofídicos (Araújo et al. 2003), alguns estudos vem revelando clara relação entre os meses com mais picos de acidentes e a pluviosidade, período de cheia dos rios e as atividades econômicas como extrativismo e agricultura (Nascimento 2000; Moreno et al.
2005; Lima et al. 2009; Waldez e Vogt 2010). As serpentes na Amazônia apresentam maior atividade durante os meses com maior pluviosidade (Martins e Oliveira 1998; Bernarde e Abe 2006), incluindo Bothrops atrox e Bothriopsis bilineata (Oliveira e Martins 2002; Turci et al. 2009) responsáveis pelos acidentes botrópicos. Nesse estudo foi demonstrado que houve um predomínio dos acidentes durante os meses mais chuvosos, estando provavelmente relacionado com aumento da atividade das serpentes e dos lavradores. Além disso, nesse período, as serpentes procuram por áreas de terra firme durante as cheias, fatores que devem aumentar a possibilidade de encontros entre humanos e esses animais.

O perfil epidemiológico das vítimas aqui observado corrobora com os demais estudos (Bochner e Struchiner 2003; Moreno et al. 2005), tendo como principais vítimas indivíduos adultos do gênero masculino em área rural, tendo como a regiâo anatômica mais atingida os membros inferiores. Apesar da maioria dos acidentes ter ocorrido em área rural, vale ressaltar que $26 \%$ deles ocorreram na cidade (zonas urbana e peri-urbana), já que algumas serpentes peçonhentas são encontradas em área urbana (e.g. Carvalho e Nogueira 1998). $\mathrm{Na}$ cidade de Cruzeiro do Sul já foram registradas Bothrops atrox, Micrurus remotus e $M$. surinamensis. Uma grande parte das vítimas (27\%) demorou mais de 24 horas para chegar ao hospital, fator que pode agravar o acidente uma vez que existe uma associação entre o tempo entre a picada e o atendimento médico com a letalidade e o agravamento do quadro (Ribeiro et al. 1998; França e Maláque 2003).

De acordo com o Manual de Diagnóstico e Tratamento de Acidentes por Animais Peçonhentos a soroterapia recomendada para acidentes botrópicos classificados como leves é de 2 a 4 ampolas, moderados de 4 a 8 ampolas e graves, 12 ampolas (França e Maláque 2003). O acidente laquético é classificado em moderado ou grave, sendo preconizado pelo Ministério da Saúde a administraçáo de 10 a 20 ampolas de soro antilaquético ou antibotrópico-laquético (Málaque e França 2003). Nesse estudo foi observado que ocorreram casos de subdosagem e também de superdosagem na administração do soro antibotrópico. De 95 casos registrados como laquéticos, seis foram considerados leves e dos 89 considerados moderados (88 casos) ou graves (um caso), apenas cinco casos receberam 10 ou mais ampolas. $\mathrm{O}$ emprego inadequado da soroterapia também foi observado no interior do Amazonas (Borges et al. 1999) e em Rio Branco no Acre (Moreno et al. 2005).

Assim como outros estudos em outras regióes na Amazônia, foi observado um despreparo no diagnóstico da serpente causadora e na conduta referente à soroterapia pelos profissionais de saúde. Tomando como exemplo a região do Alto Juruá, como conclusão, recomenda-se fortemente um treinamento desses profissionais para lidar com o ofidismo. 


\section{AGRADECIMENTOS}

Agradecemos ao Hospital Regional do Juruá, em especial Dr. Marcos Roberto de Melo Lima (Diretor Técnico do Hospital Regional do Juruá), Dr. Reginaldo Brandão dos Santos (Diretor Clínico), Irmã Lúcia Maria Lens (Gerente de Enfermagem) e Milena Lopez (Coordenadora da Vigilância Epidemiológica Municipal de Cruzeiro do Sul) pela cessáo das fichas de notificação de acidentes ofídicos. A Kleynianne Medeiros de Mendonça Costa e Reginaldo Assêncio Machado pelas sugestóes. Aos dois revisores anônimos da Acta Amazonica pelas sugestôes. Ao CNPq pela bolsa de produtividade em pesquisa para PSB (501927/2009-3).

\section{BIBLIOGRAFIA CITADA}

Almeida, M.W.B. 2004. Rights to the forest and environmentalism: rubber-tappers and their fights. Revista Brasileira de Ciências Sociais, 19: 33-52 (in Portuguese, with abstract in English).

Araújo, F.A.A.; Santalúcia, M; Cabral, R.F. 2003. Epidemioloy of accidents by venomous animals, p. 6-12. In: Cardoso, J.L.C.; França O.S.F.; Wen, F.H.; Málaque, C.M.S.; Haddad Jr, V. (Orgs). Venomous animals in Brazil: biology, clinical and therapeutic of accidents. Sarvier, São Paulo (in Portuguese).

Bernarde, P.S.; Abe, A.S. 2006. A snake community at Espigão do Oeste, Rondônia, Southwestern Amazon, Brazil. South American Journal of Herpetology, 1: 102-13.

Bérnils, R.S. (Org.). 2010. Brazilian reptiles - List of species. Available in http://www.sbherpetologia.org.br/. Sociedade Brasileira de Herpetologia. Acess in 05/11/2010.

Bochner, R.; Struchiner, C. J. 2003. Snake bite epidemiology in the last 100 years in Brazil: a review. Cadernos de Saúde Pública, 19: 7-16 (in Portuguese, with abstract in English).

Borges, C.C.; Sadahiro, M.; Santos, M.C. 1999. Epidemiological and clinical aspects of snake accidentes in the municipalities of the State of Amazonas, Brazil. Revista da Sociedade Brasileira de Medicina Tropical, 32: 637-646 (in Portuguese, with abstract in English).

Campbell, J.A.; Lamar, W.W. 2004. The Venomous Reptiles of Latin América. Comstock Publishing/Cornell University Press, Ithaca. $870 \mathrm{pp}$.

Carvalho, M.A.; Nogueira, F. 1998. Snakes from the urban area of Cuiabá, Mato Grosso: ecological aspects and associated snakebites. Cadernos de Saúde Pública, 14: 753-763 (in Portuguese, with abstract in English).

Cunha, O.R; Nascimento, F.P. 1993. Ophidians of Amazon. The snakes of Eastern Pará region. Boletim do Museu Paraense Emílio Goeldi, Série Zoologia, 9: 1-191 (in Portuguese, with abstract in English).

Fenwick, A.M.; Gutberlet Jr, R.L.; Evans, J.A.; Parkinson, C.L. 2009. Morphological and molecular evidence for phylogeny and classification of South American pitvipers, genera Bothrops, Bothriopsis, and Bothrocophias (Serpentes: Viperidae). Zoological Journal of the Linnean Society; 156: 617-40.
França, F.O.S.; Málaque, C.M.S. 2003. Botropic accident, p. 72-86. In: Cardoso, J.L.C.; França O.S.F.; Wen, F.H.; Málaque, C.M.S.; Haddad Jr, V. (Orgs). Venomous animals in Brazil: biology, clinical and therapeutic of accidents. Sarvier, São Paulo (in Portuguese).

IBGE. 2010. Demographic cense. Available in www.ibge.gov.br. Acess in 05/11/2010. (In Portuguese).

Jorge-da-Silva Jr, N. 1993. The snakes from Samuel hydroeletric power plant and vicinity, Rondônia, Brasil. Herpetological Natural History, 1: 37-86.

Lima, A.C.S.F.; Campos, C.E.C.; Ribeiro, J.R. 2009. Epidemiological profile of snake poisoning accidents in the State of Amapá. Revista da Sociedade Brasileira de Medicina Tropical, 42: 329-35 (in Portuguese, with abstract in English).

Málaque, C.M.S.; França, F.O.S. 2003. Laquetic accident, p. 87-90. In: Cardoso, J.L.C.; França O.S.F.; Wen, F.H.; Málaque, C.M.S.; Haddad Jr, V. (Orgs). Venomous animals in Brazil: biology, clinical and therapeutic of accidents. Sarvier, Sáo Paulo (in Portuguese).

Martins, M; Oliveira, M.E. 1998. Natural history of snakes in forests of the Manaus region, Central Amazonia, Brazil. Herpetological Natural History, 6: 78-150.

Melgarejo, A.R. 2003. Venomous snakes of Brazil, p. 33-61. In: Cardoso, J.L.C.; França O.S.F.; Wen, F.H.; Málaque, C.M.S.; Haddad Jr, V. (Orgs). Venomous animals in Brazil: biology, clinical and therapeutic of accidents. Sarvier, São Paulo (in Portuguese).

Moreno, E.; Queiroz-Andrade, M.; Lira-da-Silva, R.M. 2005. Clinical and epidemiological characteristics of snakebites in Rio Branco, Acre. Revista da Sociedade Brasileira de Medicina Tropical, 38: 15-21 (in Portuguese, with abstract in English).

Nascimento, S.P. 2000. Epidemiological characteristics of snakebites in the State of Roraima, Brazil, 1992-1998. Cadernos de Saúde Pública 2000, 16: 271-6 (in Portuguese, with abstract in English).

Oliveira, M.E.; Martins, M. 2002. When and where to find a pitviper: activity patterns and habitat use of the lancehead, Bothrops atrox, in central Amazonia, Brazil. Herpetological Natural History, 8: 101-10.

Pardal, P.P.O.; Rezende, M.B.; Dourado, H.V. 1997. Snakebites, p. 805-811. In: Leão, R.N.Q. (Coord.). Infectious and parasitic diseases - Focus on the Amazon. Editora CEJUP, UEPA, Inst. Evandro Chagas, Belém. 886p. (in Portuguese).

Pierini, S.V.; Warrell, D.A.; De Paulo, A.; Theakston, R.D.G. 1996. high incidence of bites and stings by snakes and other animals among rubber tappers and amazonian indians of the Juruá Valley, Acre state, Brazil. Toxicon, 34: 225-236.

Ribeiro, A.G. 1977. The climate of Acre state. Boletim Geográfico, 35: 112-141 (in Portuguese, with abstract in English).

Ribeiro, L.A.; Albuquerque, M.J.; Campos, V.A.F.P.; Katz, G.; Takaoka, N.I.; Lebrão, M.L.; Jorge, M.T. 1998. Obits by venomous snakes in the State of São Paulo: evaluation of 43 cases from 1988 to 1993. Revista da Associação Médica Brasileira, 44: 312-318 (in Portuguese, with abstract in English).

Santos, M.C. 2003. Venomous snakes and ophidism in Amazon, p. 115-125. In: Cardoso, J.L.C.; França O.S.F.; Wen, F.H.; Málaque, C.M.S.; Haddad Jr, V. (Orgs). Venomous animals in 
Brazil: biology, clinical and therapeutic of accidents. Sarvier, São Paulo (in Portuguese).

Silva, R.G.; Ribeiro, C.G. 2004. Analysis of environmental degradation in the Western Amazon: a case study of the municipalities of Acre. Revista de Economia e Sociologia Rural. 42:91-110 (in Portuguese, with abstract in English).

Turci, L.C.B.; Albuquerque, S.; Bernarde, P.S.; Miranda, D.B. 2009. Activity, habitat use, and behavior of the Bothriopsis bilineatus and of the Bothrops atrox (Serpentes: Viperidae) in Moa river forest, Acre - Brazil. Biota Neotropica, 9: 197-206 (in Portuguese, with abstract in English).

Waldez, F.; Vogt, R. C. 2009. Ecological and epidemiological aspects of snakebites in riversidade communities of the lower Purus River, Amazonas, Brazil. Acta Amazonica, 39: 681-692 (in Portuguese, with abstract in English).

Recebido em 11/11/2010

Aceito em 24/01/2011 
